\title{
The tinnitus total remission: targeting treatment to the etiological hypothesis
}

\begin{abstract}
Even with the dramatic increase in the scientific publications addressing tinnitus as the main subject, there is still some disbelief about the possibility of some treatments resulting in total and long lasting remission of the symptom. Nevertheless, sometimes the suppression of tinnitus may be observed in clinical practice after certain treatments. The objective of this study was to report a didactic case where the early adoption of therapeutic approaches targeting the initial aetiological hypotheses (metabolic and somatosensory tinnitus) ended up in a long term, total remission of tinnitus for 4.5 years until now. This illustrative case report may be a starting point for future research, such as finding the common characteristics of other individuals who have reached total remission. We recommend that a detail oriented medical and audiological evaluation of patients with tinnitus be performed to optimize treatment targeted to certain subtypes of tinnitus patients.
\end{abstract}

Keywords: tinnitus remission, suppression, treatment
Volume 10 Issue 3 - 2018

\author{
Caroline da Cunha Alves Valim,' Tanit Ganz \\ Sanchez ${ }^{2}$ \\ 'Graduate Student in Rehabilitation Sciences at the Faculty of \\ Medicine of the University of São Paulo (FMUSP) and Fellow in \\ Cochlear Implants at the Hospital das Clínicas, Brazil \\ ${ }^{2}$ Associate Professor, Otolaryngology Department, University \\ of São Paulo Medical School, Founder and Director of Ganz \\ Sanchez Institute, Brazil
}

Correspondence: Tanit Ganz Sanchez, Faculty of Medicine of the University of São Paulo/Ganz Sanchez Institute, Ganz Sanchez Institute: No. 353, Av, Padre Pereira de Andrade, São Paulo 05469-000, Brazil, Tel +55-I I-02 I525 I, Fax +55-I I-302 I525I,Email tanitsanchez@gmail.com

Received: April 20, 2018 | Published: June 12, 2018

\section{Introduction}

Tinnitus is a heterogeneous symptom regarding types, causes and degrees of severity. Few treatments have been consistently efficient. They usually focus on recovering the abnormal neural activity in central auditory pathways-a common finding in tinnitus - or quality of life, but not on aetiology. ${ }^{1}$

Although it is undeniable that treatments may benefit patients, literature lacks studies focusing on total remission of tinnitus, which may account for some disbelief about a cure. Studies that mention this phenomenon were related to significant or total recovery of sudden hearing loss when corticosteroids were prescribed within 48 hours $^{2}$ or 10 days $^{3}$ after the appearance of symptoms.

However, clinical practice and the internet show that total remission of tinnitus may somehow occur. Assuming that reaching a consensus through studies with high evidence levels is a slow process, looking deeper into the first case reports may help direct future studies.

The Brazilian Association of Otorhinolaryngology has published guidlines on diagnosing and treating tinnitus ${ }^{4}$. In alignment, our paper reports a case which reached total tinnitus remission, aiming to discuss:

i. Therapeutic approaches that target aetiological hypotheses;

ii. The total remission of tinnitus.

\section{Case report}

\section{Clinical evaluation}

ROR, 38 year-old female, attended the first consultation (August $12^{\text {th }}, 2013$ ) complaining of constant tinnitus in the right ear for 40 days, similar to an engine. She sought medical care and was told that her ears and audiometry were normal. She was prescribed oral steroids for 10 days without improvement. Then, ROR sought our services.
The medical and audiological protocol that directed to the diagnosis $^{5}$ of tinnitus made clear that ROR also had: ipsilateral constant hearing loss, constant aural fullness, hypersensitivity to sounds, frequent pain in the ears, head and neck, daily and compulsive consumption of sweets.

Annoyance was evaluated by Visual Analogue Scale (0-10) as 7 (tinnitus), 6 (hypersensitivity to sounds), 4 (hearing loss) and 3 (aural fullness).

During physical examination, she felt pain in the right temporomandibular joint and middle portion of sternocleidomastoid muscles. Blood tests (count blood cell, fasting glucose, cholesterol, triglycerides, TSH/free T4) ${ }^{4}$ were normal, as well as electrocochleography, brainstem evoked potentials and tomography of temporal bones.

The audiometry brought from another examination showed bilateral normal thresholds with asymmetry at $250 \mathrm{~Hz}, 6000 \mathrm{~Hz}$ and $8000 \mathrm{~Hz}$. We expanded the audiological battery to evaluate all symptoms (Figure 1): 1) pure tone audiometry $(250-16,000 \mathrm{~Hz})$ : asymmetry in nine frequencies, even with thresholds $\leq 25 \mathrm{dBNA} ; 2$ ) Pitch and loudness matching: $250 \mathrm{~Hz}$, narrow band noise, $17 \mathrm{dBNS}$ (average 5-10dBNS); 3) Loudness Discomfort Levels: 80-100dBNA in both ears (normal $\geq 95 \mathrm{dBNA}$ ).

\section{Diagnostic hypotheses and targeted therapy}

The following aetiological hypotheses were made:

i. Metabolic tinnitus: this subtype of tinnitus is caused or influenced by eating habits like prolonged fasting or sugar/carbohydrate abuse. The supporting findings were: presence of low frequency tinnitus $(250 \mathrm{~Hz})$ in alignment with increased threshold in this frequency, aural fullness and daily compulsive consumption of sweets; 
ii. Somatosensory tinnitus: this subtype of tinnitus is caused or influenced by dysfunction of the temporomandibular joint and/or cervical spine, or pitch/loudness modulation during movements or pain. The supporting findings were: pain in ears, head and neck, pain during palpation of the right temporomandibular joint and sternocleidomastoid muscles.

The following actions were suggested for 30 days:

a. Restricted consumption of sweets and simple carbohydrates (bread, rice, potato and pasta);

b. Temporomandibular joint evaluation: as the result was normal and her dentist did not consider further interventions, we prescribed muscle relaxant (cyclobenzaprine 5mg).

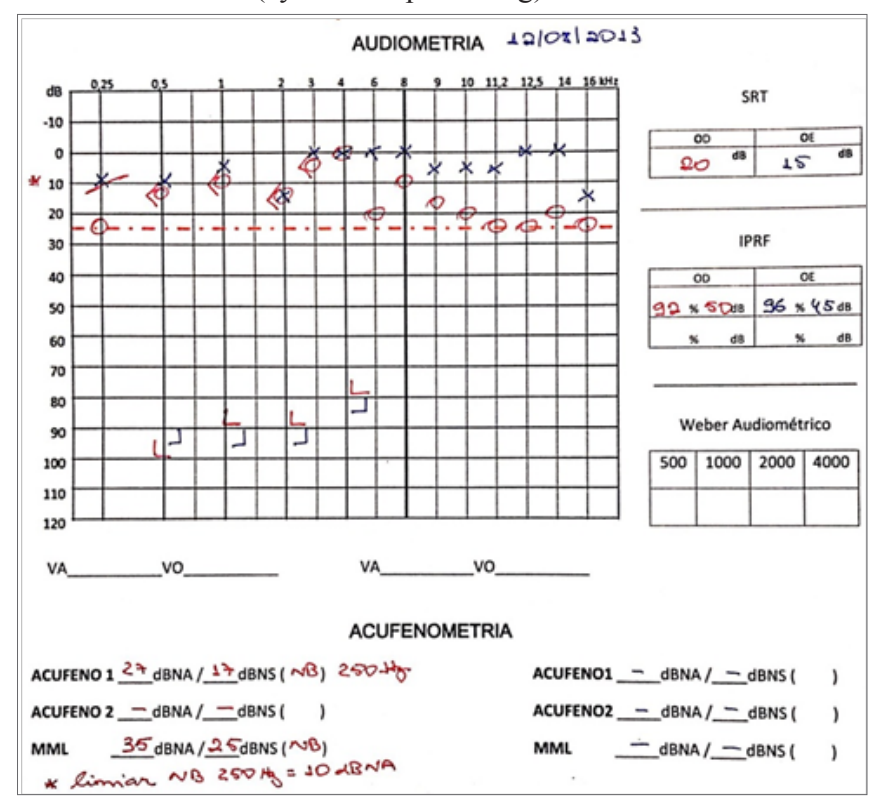

Figure I Expanded audiological battery before treatment.

\section{The total remission of tinnitus}

At the second consultation (September $13^{\text {th }}, 2013$ ), ROR mentioned four phases: 1) initial improvement for ten days; 2) interruption of the treatments and recurrence of tinnitus after two days; 3) resumption of treatments with gradual improvement over two weeks; 4) total remission of tinnitus in the last week. To confirm such remission, she was placed inside the acoustic chamber to enhance selective auditory attention, and no tinnitus was noticed. Due to the excellent result, ROR was instructed to stop medication and to adopt low consumption of simple carbohydrates and sweets.

On the third visit (November 11 $1^{\text {th }}, 2013$ ), ROR reported that she had been in total remission of tinnitus for the last nine weeks. She also noticed gradual improvement of hearing loss and aural fullness. Sound intolerance had partial improvement. The audiometry performed at this time showed recovery of correct thresholds (Figure 2).

The patient has been monitored annually. During the last phone call (March $8^{\text {th }}, 2018$ ), she reported remaining asymptomatic since September 2013, confirming the stability of total remission of tinnitus in the period of four years and six months, so far.

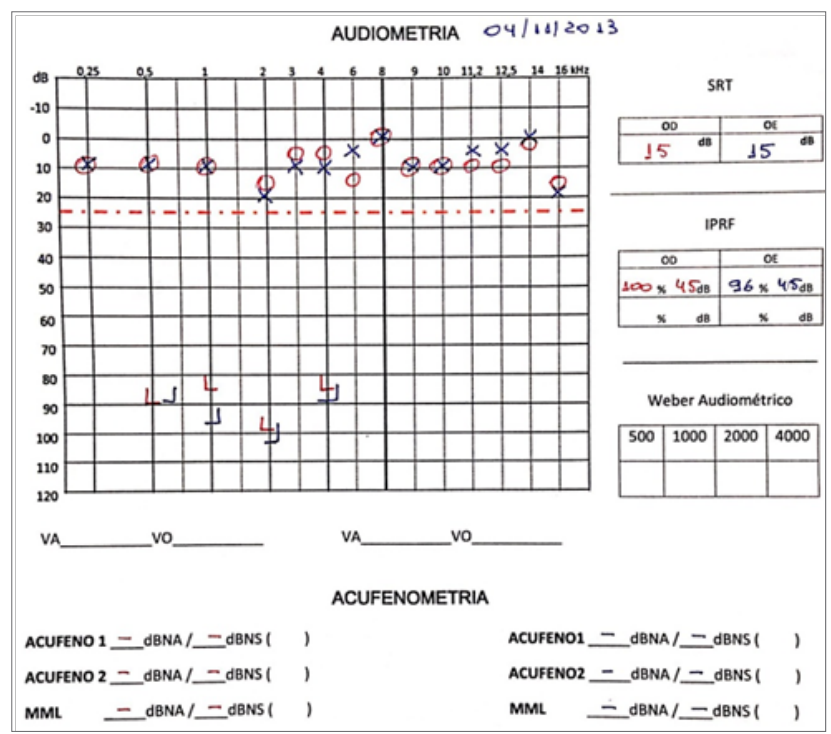

Figure 2 Expanded audiological battery after treatment.

\section{Discussion}

Older studies have focused predominantly on peripheral auditory lesions as the main trigger for the onset of tinnitus. Newer studies have highlighted the reorganization of central auditory pathway connections among multiple areas, even though they still mention that the onset of tinnitus is subsequent to damage in peripheral auditory pathways. Thus, the therapeutic approach focused on ear recovery gave rise to different options focused exclusively on reorganizing the abnormal electrical impulses in the brain, sometimes ignoring the ear.

We agree that the plastic changes occurring at different levels of the central pathways determine the persistence of tinnitus and its severity. However, we believe that the approach focused on the correction of cochlear alterations is still worth it, especially when performed at early stages, because recovering the ear-whenever possible-would prevent progression of neuroplastic changes towards the central auditory pathways.

Our patient sought early medical help, but prescription of corticosteroids without a clear diagnosis failed in promoting improvement. This conduct reflects a non-specific approach. Although it is not easy to define the tinnitus aetiology at the first consultation, at least the main hypotheses should be clarified to help choose the targeted treatment.

As mentioned before, ROR received two aetiological hypotheses based on four items: the detailed anamnesis, physical exam, normal electrophysiological/imaging tests and high frequency audiometry showing the extension of the damage in the right ear. It is pertinent to discuss the effect that thresholds "within normal limits" have on professionals, since patients are commonly told that they have nothing in the ear. In our case, the conventional audiometry, an instrument that most professionals have access to, already showed asymmetry in $250 \mathrm{~Hz}, 6000 \mathrm{~Hz}$ and $8000 \mathrm{~Hz}$. Associating this finding with the consistent complaint of right hearing loss, such asymmetry seems abnormal. In addition, visualization of the asymmetry between 
$9000 \mathrm{~Hz}$ and $16000 \mathrm{~Hz}$ confirmed the aggressive damage to several cochlear regions, although thresholds did not exceed 25dBNA.

Following the rationale of the possible aetiologies, we suggested the correction of dietary habits for the metabolic hypothesis, as it is indicated for sugar-compulsive patients who undergo hypo/hyperglycemia or hyperinsulinemia. ${ }^{4,6}$ Assessment of the temporomandibular joint and cervical muscles was the initial strategy for the somatosensory hypothesis. As it was normal, we prescribed muscle relaxant. ${ }^{7}$

\section{Outcomes of long-term total remission}

Some aetiologies of tinnitus may be overlapped in a single subject (as in ROR), which limits the likelihood of a single treatment succeeding in all patients. It was not our intention to evaluate efficiency of a diet or medication, but to show that specific approaches targeting the aetiologies may succeed.

Negative ideas about tinnitus ("it has no cure", "there is nothing to be done" and "learn to live with it") are often exposed. Most professionals and sufferers do not believe in the possibility of total remission because science does not show it. Randomized controlled trials (RCTs) are gold standards to evaluate treatment. As they have shown few efficient treatments, patients tend to be overlooked. Two clinical arguments may imply the difficulty of certain treatments to overcome placebo:

a. Patients may take longer to respond significantly to certain treatments and RCTs often observe the short term effect.

b. Many RCTs are designed without subtyping tinnitus according to some criteria (for example: metabolic, somatosensory, pulsatile, myoclonic etc). ${ }^{8}$ Thus, heterogeneous patients are treated homogeneously. Evaluating the effect of a diet may differ if the study group contains more or less patients with metabolic tinnitus included without prior evaluation. Likewise, the effect of a muscle relaxant may also differ if study groups contain more or less patients with somatosensory tinnitus.

The natural diversity of tinnitus makes it unlikely that RCTs display total remission of tinnitus, but people who consider themselves cured of tinnitus do exist, suggesting that the knowledge generated by research and by clinical practice does not match.

Our patient achieved total and long lasting remission of tinnitus up to the last follow-up. Although opinions may diverge, we consider that such an excellent result was obtained from the approaches targeted to the initial diagnostic hypotheses, since no benefit was obtained with the nonspecific approach that she had received before. Illustrative case reports about total remission may be a relevant starting point for future research, such as finding the common characteristics of such individuals.

\section{Conclusion}

The patient achieved total remission of tinnitus for 4.5 years after receiving treatments focused on the main diagnostic hypotheses defined in the first consultation. We recommend that a detail oriented medical and audiological evaluation of patients with tinnitus be performed to optimize treatment targeted to certain subtypes of tinnitus patients.

\section{Acknowledgements}

The authors would like to express our very great appreciation to the patient ROR for clarifications about the details of the process of tinnitus total remission.

\section{Conflict of interest}

The author declares that they have no conflict of interest.

\section{Ethical approval}

For this type of study formal consent is not required.

\section{References}

1. Baguley D, McFerran D, Hall D. Tinnitus. The Lancet. 2013; 382(9904):1600-1607.

2. Mühlmeier G, Baguley D, Cox T, et al. Characteristics and Spontaneous Recovery of Tinnitus Related to Idiopathic Sudden Sensorineural Hearing Loss. Otol Neurotol. 2016;37(6):634-41.

3. Jung AR, Kim MG, Kim SS, et al. Clinical characteristics and prognosis of low frequency sensorineural hearing loss without vertigo. Acta Otolaryngol. 2016;136(2):159-63.

4. Onishi ET, Coelho CCB, Oiticica J, et al. Tinnitus and sound intolerance: evidence and experience of a Brazilian group. Braz J Otorhinolaryngol. 2018;84(2):135-149.

5. Sanchez TG. Zumbido. Tratado de Otologia. In: Bento RF, Miniti A Marone SAM, editors. São Paulo, SP: Editora USP; 1998:322-330.

6. Li J, Zhang Y, Fu X, et al. Alteration of auditory function in type 2 diabetic and pre-diabetic patients. Acta Otolaryngol. 2018;181-6.

7. Vanneste S, Figueiredo R, De Ridder D. Treatment of tinnitus with cyclobenzaprine: an open-label study. Int $J$ Clin Pharmacol Ther. 2012;50(5):338-44.

8. Dobie RA. A review of randomized clinical trials in tinnitus. Laryngoscope. 1999;109(8):1202-1211. 